\title{
The Relation of the Heart-Beat to Electrolytes and its Bearing on Comparative Physiology.
}

\author{
By
}

George Ralph Mines,

Fellow of Sidney Sussex College, Cambridge.

As objection often raised to experiments on tissues treated with artificial solutions and isolated from nervous control is that results obtained under such highly abnormal conditions can be of no real value. This criticism arises essentially from a misconception as to the aim of the experiments in question, but it is encountered so frequently that it may not be out of place to consider briefly why it is necessary for the physiologist who would learn something of the more intimate mechanism of the cell to work with tissues under artificial conditions.

Let us suppose that the action of some chemical substance on the heart-beat is the subject of investigation. It is found, we will suppose, that on injecting a solution of the substance into the circulation of an intact animal the beat of the heart is modified in some way-it may be in frequency or force, or in the rhythmic sequence of its chambers. If on repetition of this simple experiment, made on animals as far as possible under normal conditions, the same result is consistently obtained, the result may be of therapeutic interest. For its interpretation in terms of what is already established about the mechanism of the body, a searching physiological analysis is, however, required. The effect on the heart may be direct or it may be due to one of a variety of indirect actions of the substance.

Thus, for instance, it may be due to an action of the substance-

(1) on sensory nerve endings connected to the cardio-inhibitory or cardio-accelerator centres in the medulla;

(2) on the nerve cells in these centres;

(3) on cell stations in the course of the vagus or sympathetic nerves to the heart;

(4) on the respiratory movements, modification of which affects the condition of the blood in such a way as to influence the heart, either directly or through action on the nerve centres which can control it; 
(5) through an alteration in the blood pressure (which may affect the heart by a local or a central point of attack) due to constriction or dilatation of arterioles, this being caused either by reflex, central or local action;

(6) on the heart muscle itself.

By an elaborate series of experiments, involving the section of nerves, the use of drugs such as atropine and curare, the enclosure of organs in plethysmographic apparatus; by taking records of blood pressure and by ventilating the lungs steadily with the aid of a mechanicallydriven respiration pump, it would be possible to determine by which of the various possible means the effect of the injected substance on the heart was produced.

Suppose, now, that by such experiments the point of action of the substance has been traced to the heart muscle itself. (Be it noted that the elucidation of this point has involved the imposition of more and more artificial conditions.)

The experiment remains of comparatively little value to the physiologist, who is endeavouring, by the investigation of the effects of substances on the activity of the heart, to learn more about its actual mechanism. For the experiment can only in the vaguest sense be called quantitative. However carefully the dose of the drug is weighed out, the concentration of the substance in the blood reaching the heart cannot be known at all accurately, since the dilution depends not only on the rate at which the injection is made and on the rate of flow in the vein into which the injection is made, but also on the blood flow in all the other veins leading to the heart. Moreover, it is seldom justifiable to assume that the injected substance remains unchanged in the blood. Blood is a highly complex fluid: the carbonates, phosphates, and in particular the proteins present in it, enter into chemical or adsorptive relation with many injected substances. Of course, from a therapeutic standpoint this does not matter. If, for instance, the injection of a particular drug is followed by marked strengthening of the heart-beat, it matters little to the practitioner whether the substance actually presented to the heart as a result of his injection is the same chemical substance as that which left the hypodermic syringe, or a product of some complex reaction between that substance and the blood or tissue fluids. But when the object is not simply the production of the effect, but the explanation of how it is produced; when the response of living cells to changes in their chemical environment is being used to throw light on the mechanism and properties of the tissues themselves, it is clearly of the first importance that the change 
in the environment in any experiment should be known with the utmost precision.

Practically every experiment involves the imposition of some abnormal conditions. In a state of nature, conditions are varying continually; the fixation of any condition in itself constitutes an abnormality. After determining artificially one condition, it is fallacious to speak of the remaining conditions as being "normal." If they too are not experimentally controlled, they are unknown. The choice lies, therefore, between a type of experiment in which most of the conditions are unknown but where there has been but little operative interference, and another type where the conditions are simplified and controlled by artificial means. The former may yield information of much value to the physician, but it is to the latter that we must turn for advances in our knowledge of cell physiology.

If, now, we are concerned with the problem of how and why the heart gives rhythmic contractions, the first step will be to determine and to define the simplest conditions under which the heart will continue to beat. Removal of the heart from a freshly killed animal suffices to show that the essential mechanism is self-contained. The movements of the heart, though susceptible of control by the central nervous system, are yet able to continue when all nervous connection is severed, and indeed it is known that in the chick they begin in the heart muscle some time before any nervous connection is established. It might be suggested that the excised heart lying in a watch-glass and continuing to beat was under the simplest conditions imaginable. Very little consideration is needed to show that this is not the case. In the first place, its temperature is not being controlled; and secondly, the heart muscle is in contact with a layer of fluid of complex and changing composition. As so often in scientific work, an elaboration of apparatus is necessary in order to secure a simplification of experimental conditions. That the composition of the fluid in contact with the muscle may be known as closely as possible it is desirable that a large volume of the fluid should be available, and that the portion in contact with the tissue shall be frequently renewed. For this purpose it is not sufficient to immerse the heart, or portions of it, in a large vessel of the solution. Such a method, it is true, has often been adopted, but it is far from being satisfactory. The best method is to perfuse the heart with fluid, letting the solution enter the venous end of the heart at a small and constant pressure, and allowing it to escape at the aortae, which may be opened up to allow of a free flow. In this way, in the hearts of animals, where the coronary system has attained to no 
great development,* the heart muscle, and in particular that of the thin-walled auricles and sinus venosus, is brought into very intimate relation with a constantly renewed layer of fluid. The stream of fluid may be said to control the temperature and the chemical environment of the cells. The efficiency of the perfusion method is shown by the fact that with a suitable fluid the heart may be kept beating regularly and strongly for many hours, while slight changes in the composition of the perfused fluid with respect to an important ingredient are followed by evident change in the character of the beats within a very few seconds.

As might be expected, the blood or serum from the animal whose heart forms the subject of experiment forms a perfusion fluid effective in maintaining the activity of the heart. But the classical researches of Ringer revealed the all-important fact that these highly complex, protein-containing solutions could be satisfactorily replaced (in the case of the frog's heart) by certain solutions of simple inorganic salts. T\} e presence of the inorganic salts of the serum in the fluid in contact with the cells of the heart muscle is of more immediate importance for the activity of these cells than is the presence of any organic food-stuffs. It is true that it has been shown that the addition to the salt solution of a small amount of glucose keeps'the heart going for longer periods than are attained without it; and according to some the addition of proteins has a beneficial effect, though this is denied by others who have studied the point. The fact remains that with a solution of simple inorganic salts in distilled water containing some dissolved oxygen the hearts of various animals can be kept beating outside the body for very many hours. $\dagger$

During this time, while the heart continues to contract regularly and with sufficient force to enable it to pump through fluid with such energy that if it were still connected to the arteries it could fulfil its normal function of propelling fluid through the vascular system, and while the frequency and form of the contractions remain essentially like those observed in the heart of the uninjured animal, we may say that the heart muscle is exercising its normal function, and although the conditions are frankly abnormal in many respects, yet they embrace those factors of environment which are immediately necessary for the normal behaviour of the cardiac muscle cells.

We must next consider what are the essential constituents of a salt solution which will answer this purpose.

* In mammalian hearts it is necessary to perfuse the coronary system.

† In working with the hearts of Elasmobranchs, as Baglioni (Zentralbl. f. Physiol. 19, 385, 1905 ) showed, it is necessary to add urea to a neutral perfusion fluid. 
The "Ringer's solution" most commonly employed in the physiological laboratory contains chiefly sodium chloride with small quantities of potassium and calcium chlorides and of sodium hydrogen carbonate. The actual concentrations of the salts employed differ according to the animal used; for the frog the total concentrations should be about $\cdot 125 \mathrm{Mol}$. If the concentration of sodium chloride be called 100 the relative concentrations of the other salts may be taken as 2.5 of potassium chloride, 2 of calcium chloride, and 1.5 of sodium hydrogen carbonate. This solution, simplified by the omission of the last-named ingredient, still maintains the heart-beat in a satisfactory manner. We shall return in a moment to the function of the sodium bicarbonate. Its presence is not essential. Removal of the potassium salt leads to an alteration in the character of the beats, the systolic phase becoming prolonged, but does not necessarily cause arrest or even weakening of the beats. With a solution consisting only of distilled water with sodium and calcium chlorides and some dissolved oxygen, if the concentration of the $\mathrm{Ca}$ is carefully adjusted, the frog's heart may be kept beating vigorously for prolonged periods.

Removal of the calcium from the perfusion fluid is immediately followed by great weakening of the contractions, which in the course of a few minutes become exceedingly feeble and before long cease altogether, the heart remaining in a relaxed state. When the calcium is replaced the beats start again at once and become as vigorous as at the start. In the frog, as Ringer showed, the calcium may effectively be replaced by strontium. It may also be replaced by barium, but only to a very limited extent, for in a short time barium causes arrest of the heart in a firmly contracted state.

It should be noted that practically any soluble salt of calcium may be used in place of the chloride. The ionic theory is peculiarly acceptable to the physiologist in that it gives concrete expression to the fact so often encountered, as here, that all solutions of a particular metal, where on physico-chemical theory it should be in the same ionic condition, present in common certain definite physiological actions. We may say that the calcium ion is essential for the activity of the heart. There is reason to think that the calcium forms with some constituent of the heart muscle a readily dissociated compound, whose presence is a cardinal factor in the activity of heart muscle. The closely similar strontium ion can form a compound so similar to the calcium compound that it can fill the same niche in the architecture of the muscle, while the barium ion may best be pictured as forming a compound of similar composition but probably almost irreversible, perhaps through great insolubility. No metal other than these has been found to replace calcium. 
It is of interest to find that these relations of the heart muscle to $\mathrm{Ca}, \mathrm{Sr}$, and $\mathrm{Ba}$ are essentially the same, not only in other vertebrates which have been studied, but also in the heart of the mollusc Pecten.

So far then the features of immediate importance in the perfusion fluid for the heart appear to be the presence of certain concentrations of sodium and of calcium ions, the presence of dissolved oxygen and the maintenance of the temperature within certain limits.

Keeping these conditions constant, let us consider the effects of varying the composition of the fluid in certain other respects.

Since we deal always in physiological work with aqueous solutions, and since water is to a slight extent electrolytically dissociated, our solutions always contain hydrogen ions and hydroxyl ions. In pure water these are of course present in equal numbers, the concentration of each, expressed in gram-ions per litre, being at room temperature about $10^{-7}$.

It follows directly from the general principle expressed in the law of mass action that the addition to the water of any substance tending to increase the concentration of hydrogen ions will reduce the concentrations of hydroxyl ions. A similar shift in the equilibrium will be produced by the addition of any substance tending to remove hydroxyl ions. The ratio of the $\mathrm{H}^{\cdot}$ to $\mathrm{OH}^{\prime}$ is increased. An inverse change is produced by the addition of $\mathrm{OH}^{\prime}$ or the removal of $\mathrm{H}$ :

Knowing the dissociation constant of water, if the hydrogen ion concentration of any aqueous solution is given it is a matter of simple arithmetic to calculate the hydroxyl ion concentration and the ratio between the hydrogen and hydroxyl ion concentrations for that solution.

To define the position of this $\mathrm{H} \cdot, \mathrm{OH}^{\prime}$ equilibrium for any solution it is sufficient therefore to state the hydrogen ion concentration of the solution. This is expressed most conveniently as a logarithm.

To avoid the constant repetition of the phrase "hydrogen ion concentration" we may adopt Sörensen's notation in which the symbol $\mathrm{P}+$ is followed by the logarithm of the hydrogen ion concentration, $\mathrm{H}$

the minus sign being understood. Thus describing a solution as being of $\mathrm{P}+7.07$ means "the hydrogen ion concentration of the solution is $\mathrm{H}$ equal to $10^{-7.07}$ gram ions per litre."

An aqueous solution whose $\mathrm{P}+$ at $18^{\circ} \mathrm{C}$. is 7.07 is neutral in reaction. $\mathrm{H}$

If the $\mathrm{P}+$ is numerically less than 7.07 the solution is acid, if greater $\mathrm{H}$ 
it is alkaline. For instance, the $\mathrm{P}+$ of centi-normal hydrochloric acid $\mathrm{H}$

is 2 , that of milli-normal sodium hydroxide 11 , and so forth. Now the solution of pure salts of strong acids and strong bases in distilled water, neutral by intention, becomes in the course of preparation very faintly acid as it reaches equilibrium with the small concentration of $\mathrm{CO}_{2}$ present in the air. The reaction of pure water or of a solution of salts of the type we have just mentioned, which do not themselves affect the reaction, is very easily shifted by minute additions of acid or alkali. Thus one drop ( 05 c.c.) of normal hydrochloric acid would suffice to change the $\mathrm{P}+$ of fifty litres of water from 7 to 6 . But if the origin-

$\mathrm{H}$

ally neutral solution contained a weak acid balanced against a salt of the same weak acid with a strong base, the addition of the same amount of acid as before would not appreciably disturb the P+. Such

a condition is realized, for example, in a saline solution containing a little sodium bicarbonate and carbonic acid. Such a solution may be prepared with the same $\mathrm{P}+$ as the purest water: it will differ from

$\mathrm{H}$

water in that its $\mathrm{P}+$ is much more stable-that is to say, it is displaced $\mathrm{H}$

very much less by the addition of the same small traces of acid or of alkali. Substances having this effect on addition to a solution are spoken of by Sörensen as "buffers." Amphoteric electrolytes such as the amino-acids and their compounds the proteins tend also to stabilize the reaction of solutions in which they are present. The explanation of the action of all these substances lies in the fact that when they are present in a solution the hydrogen ion concentration is a function of more than one equilibrium. It should be noted in passing that in nature such "buffers" play an important rôle. For instance, the carbonates, phosphates, and proteins in the blood serve to limit the changes in $\mathrm{P}+$ of that fluid resulting from the production of acid $\mathrm{H}$

metabolites by the tissues, the secretion of acid or of alkaline fluids, and the removal of carbonic acid in the lungs, to such values that the limits of safety are never passed. If the blood could be deprived of these substances, it is probable that the acid produced in moderate exercise for example would so raise the $\mathrm{H} \cdot$ concentration as completely to disorganize nerve cells and heart, instead of altering it only to such an extent as to cause the blood to act as a chemical messenger or hormone, modifying the activities of nerve cells and heart in such a 
way as to restore the normal value of the $\mathrm{P}+$ of the blood. For this is what happens in actual life.

$\mathrm{H}$

The action of acids and alkalies on the heart was described by Gaskell nearly thirty years ago. Let us note in detail the effects of acid. Gaskell used lactic acid, but the description he gave of its action on the heart applies equally to the effect of other acids, such as hydrochloric or even carbonic. The action on the heart is due to what they have in common: an excess of the hydrogen ion. The essential features of the action on the heart are these: The acid solution diminishes the force of the systole, inereases the diastole, and causes arrest of the heart in a state of complete relaxation. If now the acid solution is washed out with "neutral saline" (i.e. $\mathrm{P}+$ about 6.5 ),

$$
\mathrm{H}
$$

there is no recovery for some time, and then very feeble beats may start. But if a slightly alkaline solution is used the recovery is prompt, and the beats become as strong as at the start within a few seconds, or at most in a minute or two. Further experiment shows that the persistence of the after effect of the acid bears a definite relation to the $\mathrm{P}+$ of the acid solution and to the duration of its $\mathrm{H}$

application. With a minimal dose of acid applied for the shortest time necessary to cause arrest of the heart, recovery on perfusion with "neutral saline" may be fairly prompt and complete, but if the dose is rather greater or applied longer the effect may persist for an hour or more during subsequent perfusion with neutral saline. This is to be regarded as the typical effect of acid on the heart. If the solution is made too alkaline the heart is also stopped, but in this case in systole. In the case of the frog's heart the divergence from neutrality needed to produce the systolic alkaline arrest is much greater than that needed to produce the diastolic acid arrest. Roughly, the limits are about $\mathrm{P}+10$ and $\mathrm{P}+5.5$ respectively. It is not surprising then to $\mathrm{H} \quad \mathrm{H}$

find that "neutral saline" restores the heart from the alkaline arrest much more quickly than from the acid arrest, for this solution is much nearer the "acid" limit than the "alkaline" limit.

So far then we have these facts. The normal activity of heart muscle (by which we mean its power of giving automatic rhythmic contractions) is possible only when the hydrogen ion concentration of the solution in contact with it lies within certain limits. If these limits are considerably exceeded on the acid side, not only is the heart stopped, but some alteration. is impressed on the muscle, which is removed only very slowly, if at all, by a "neutral" solution, but instantly by an alkaline solution.

* The slight deviation from strict neutrality is due to $\mathrm{CO}_{2}$ from the air. It is convenient to use such a solution and it will maintain the heart-beat for hours. 
In working at the action of salts on the heart I was led, for reasons which need not be entered into here, to investigate the action of salts of the rare earth metals such as lanthanum, yttrium, neo-dymium-in all eleven were studied.

These salts in aqueous solution yield trivalent ions, La.. $\mathrm{Nd} \cdots$, etc. Unlike the salts of other metals yielding simple trivalent ions (such as aluminium, gold, iron, etc.), these salts of the rare earths do not render the solution acid in reaction-they do not undergo hydrolytic dissociation. I found that remarkably small concentrations of these salts in "neutral" solution sufficed to stop the heart. Thus the frog's heart is invariably stopped within a few minutes, generally within thirty seconds, by a solution containing .00001 gram molecules per litre of either of these rare earths, and I have often seen it stopped by a concentration of one-millionth molecular. I find the same action on the heart of the rabbit and of the ray as on that of the frog.

Studied in detail on the frog's heart, the effect of the simple trivalent ion parallels that of acid in every particular. The mode of arrest, the absence of recovery, or the slow and imperfect recovery on washing out with " neutral saline," and the immediate and complete recovery on the use of alkali, are all reproduced exactly.**

How are these phenomena to be interpreted?

Can we discover any physico-chemical explanation for the close similarity in their action on the heart of these ions, the hydrogen ion and the ion of the trivalent rare earth, which are so different chemically, so widely different in their mobilities and their volumes?

Let us first review very briefly what other considerations have led physiologists to infer as to the nature of that subtle mechanism by which muscular tissues like that of the heart transform chemical into mechanical energy with such high efficiency.

The recent work of $\mathrm{A}$. V. Hill on the time relations between heat

* Quantitative investigation shows that these simple trivalent ions are more powerful than the chemically equivalent concentration of hydrogen ions in their effect on the heart. And thus it happens that if the activities of solutions yielding trivalent ions which do undergo hydrolytic dissociation are compared with those which do not, the latter are found more powerful in their action on the heart. For instance, taking equimolecular solutions of aluminium, scandium, and lanthanum chlorides, the $\mathrm{Al} \cdots$ liberated in the first solution combines to some extent with the $\mathrm{OH}^{\prime}$ in the water, forming an almost undissociated hydroxide and liberating an equivalent of $\mathrm{H} \cdot(3 \mathrm{H} \cdot$ for every $\mathrm{A} 1 \cdots)$. Thus the solution becomes acid. In the lanthanum solution there is no hydrolysis, for lanthanum hydroxide is strongly dissociated. Thus the solution remains "neutral," and contains its full complement of La $\cdots$. The case of scandium is intermediate : its solution is less acid than the A1 solution. Now it is found that while all three solutions produce the same kind of effect on the heart, the lanthanum solution is che most and the aluminium solution the least powerful of the three. 
production and shortening in muscle disproves the hypothesis that the shortening is an effect due to the action of local rise of temperature on some constituent of the muscle structure. The muscle is certainly not a heat engine.

The so-called osmotic theory of contraction-which supposes that as a result of the liberation of a high concentration of electrolytes within certain membranous compartments of a particular shape, an increase of hydrostatic pressure is set up within them causing them to bulge and shorten, thus producing the shortening of the whole muscle-meets with the objections that the osmotic pressure which would be necessary to give rise to mechanical effects of the magnitude encountered in muscular contraction would necessitate extraordinarily high concentrations of electrolytes, and such changes could scarcely be completed in the very brief interval which often suffices for the completion of a muscle twitch.

The sort of explanation which has most to recommend it is that which refers the act of shortening to a mechanism in which the alteration of surface tension at the junctions of different phases is the motive power. Anyone who has studied the behaviour of a globule of mercury, lying in a dish of dilute sulphuric acid to which a trace of potassium chromate has been added, when touched by a clean iron wire, will recognize in the sudden drawing together of the globule movements which recall more forcibly than any other artificial scheme the movements of living contractile tissue. Here, too, the evolution of a considerable amount of kinetic energy is the result of a very small amount of chemical change.

As to the mechanism by which surface tension alterations can be supposed to play a part in the muscle, there are two chief hypotheses. Both suppose that as a result of the process of excitation in the muscle there is a liberation of excess of some electrolyte in the tissue. According to one view, an ion of this electrolyte combines chemically with some protein constituent of the muscle to form a new ion-protein compound possessing different physical properties-the protein being so disposed in the muscle that an alteration in its surface tension will exert a pull on the whole structure and lead to shortening.

Another view is that the electrolyte is liberated in a system presenting surfaces possessed of differential permeability towards the ions and that thus the liberated electrolyte sets up differences of electrical potential across these surfaces resulting in mechanical strain and alteration in the contour of these surfaces. This last view, though it is at present of necessity somewhat vague, has on the whole more to recommend it than either of the others. The electrical phenomena 
which accompany the contraction as well as the excitation processes in muscle provide important evidence, and other facts might be adduced in favour of the idea that an essential part in the mechanism of the contractile process in muscle is played by surfaces or membranes possessed of differential permeability towards ions. It is familiar that such membranes form an integral part in the theories of excitation propounded by Nernst and elaborated by Lapicque and by Lucas and Hill.

The existence of membranes or surfaces in muscle is granted by the histologist to almost any desired extent. In the case of skeletal muscle indeed, it is scarcely an exaggeration to say that every histologist who has undertaken the investigation of its structure has described and attached his name to some new membrane, line or band.

It is clear then that we are not making any new or rash assumption in stating that certain surfaces or membranes form an integral part of the muscle mechanism, and the ionic permeability of these membranes * is a factor of importance in that mechanism.

The possession by a membrane of differential ionic permeability is no imaginary attribute: simple experiment shows it to be a very usual property. This fact is brought out most clearly by experiments on the influence of membranes on concentration cells.

Direct experiment shows also that the ionic permeability of membranes-and a great variety of them have been tested-is profoundly influenced by certain features of the solutions in contact with them. For a given membrane the factors likely to influence its ionic permeability are-

(1) The $\mathrm{P}+$ of the solution with which it is treated.

$\mathrm{H}$

(2) The presence in that solution of polyvalent ions. The simple trivalent positive ions are enormously more powerful than the divalent.

When a membrane such as a piece of peritoneal membrane, the lining of an egg-shell or a thin sheet of gelatine, is treated with a very small concentration of acid, or of one of the simple trivalent ions of which we have spoken, its ionic permeability is modified. Direct experiment shows that this altered condition is retained, it may be for minutes or hours, while the membrane is washed with a "neutral " solution free from the polyvalent ions-the persistence of the effect depends on the nature of the membrane, the concentration of the solution used to produce the

* Here and in the following pages we employ the word membrane with the understanding that it need not necessarily mean anything more than the surface of separation between two phases in the system. 
alteration and the length of time it was allowed to act-but if the membrane is treated with a faintly alkaline solution the effect of the acid or the trivalent ion is removed at once.

The parallel with the action of these same agencies on the heart is sufficiently striking. And the parallel does not end here.

Seeking to test further whether this potent action on the heart is indeed a general property of trivalent ions, I employed solutions of certain cobaltammines which yield complex trivalent ions in neutral solution. The compound used most frequently was luteo-cobalt chloride $\left[\mathrm{Co}\left(\mathrm{NH}_{3}\right)_{6}\right] \mathrm{Cl}_{3}$, which yields the ion $\left[\mathrm{Co}\left(\mathrm{NH}_{3}\right)_{6}\right] \cdots$. Through the generosity of Prof. Werner I was able to test also four other complex trivalent ions. All agreed in showing very much weaker action on the heart than the rare earth solutions. A concentration of the complex ion of the order of 1000 times as great as of the simple ion is needed to stop the perfused heart. A similar difference is shown in the action of these ions on the gelatine membrane. Even large concentrations have only a slight effect on the ionic permeability of a membrane of this type.

The influence of hydrogen ion concentration and of trivalent kations on the heart is paralleled in detail by the action of these ions on the ionic permeability of certain artificial membranes.

The ionic permeability of a membrane can be shown, both theoretically and experimentally, to depend-other things being equal—on the electric charge of the membrane.

Now it can be demonstrated by a variety of methods that most membranes in water, or in a solution like our "neutral saline," possess a negative charge. This charge is reversed with great ease by acid or by the rare earth solution. This can easily be shown, for instance, by the use of dyes, which are themselves charged colloids. A piece of gelatine soaked in "neutral saline" stains very feebly with eosin, strongly with methylene blue. But after treatment with acid or rare earth it takes up eosin with great avidity and is hardly tinged by methylene blue. Eosin consists of negatively charged particles, methylene blue of positively charged particles. If the membrane has a negative charge it can hold the latter, if a positive charge, the former. This method also reveals the fact that the complex trivalent ions are much less potent than the simple trivalent ions in altering the charge of the gelatine membrane.

In these membranes we are, of course, dealing with substances in the colloidal state. If instead of using extended sheets of material we turn our attention to colloidal solutions, where one phase of the colloidal system consists of discrete particles floating freely in the 
other, it is possible to study the action of electrolytes more simply still. For in such systems the alteration of electric charge on the particles is often followed by an alteration in their state of aggregation, which may be manifested as an alteration in the colour of the solution, or as is very usual by actual precipitation. For instance, when the particles are negatively charged, as in colloidal gold or in a solution of egg-white, the addition in suitable concentrations of electrolytes capable of conferring positive charges on the particles causes agglutination of these particles.

Testing a large number of colloidal solutions by this method, it becomes apparent that there are great differences in the relative efficiencies of different ions in affecting the charge of different surfaces. Thus considering the simple and the complex trivalent ions one finds that many colloidal solutions are quite as readily precipitated by them as by the simple trivalent ions (e.g. colloidal gold, arsenious sulphide, boiled diluted egg-white, and many more), while others which are readily precipitated by the simple trivalent ions are unaffected by even large concentrations of the complex ions (e.g. unboiled diluted eggwhite, haemoglobin, etc.). Of a large number of colloidal solutions, those which are very sensitive to complex as well as to simple trivalent ions are found to belong to that class of colloids known as lyophobe or suspensoid; those sensitive to the simple but insensitive to the complex trivalent ions, to the class called lyophil or emulsoid.

Clearly, what has been stated would indicate that the membranes in the heart muscle are of an emulsoid colloidal material. This indeed confirms in one special instance a conclusion drawn from entirely different considerations by Martin Fischer as to the nature of the body proteins in general. The study of the action of these electrolytes on the heart will give more precise information than this. When the ratios of the activities of the simple and the complex ions on surfaces of various compositions have been worked out in detail, we shall be enabled to define precisely some physico-chemical constants for each surface. As has been remarked, the investigations of colloid chemistry have not as yet been carried far enough for this to be done, but the methods are available, and the research would present no insuperable difficulty. Just as the classification of liquids of two phases into suspensions, colloidal solutions and true solutions, is now recognized to be an arbitrary though convenient subdivision, there being no real boundaries between the classes; so it will probably be found among the colloidal solutions themselves. We shall learn in course of time to describe each colloidal solution in terms of certain essential factors which will include such terms as the viscosity of the phases, the 
amount of surface presented by them, and the electric charge they carry. At present this cannot be done in any quantitative sense, yet we may illustrate from material already available the nature of the biological interest which will accrue from knowledge of this kind.

In the first place, new light is thrown on the relations between the living heart muscle and its normal environment.

We have considered the importance of the $\mathrm{P}+$ of the solution. $\mathrm{H}$

Take now the case of magnesium. $\mathrm{Mg} \cdots$ is the only simple divalent ion which can be expected to act on the heart simply quâ divalent ion. $\mathrm{As}$ we have shown, $\mathrm{Ca} \cdots, \mathrm{Sr} \cdot$, and $\mathrm{Ba} \cdots$ enter probably into special chemical relation with some constituent of the tissue, while the other metals which give divalent ions (such as beryllium, zinc, cadmium, etc.) form solutions which are hydrolyzed.*

It is generally stated that an emulsoid colloid is very insensitive to salts. This is not true, at any rate in the case of some typical emulsoids, when simple trivalent ions are considered. Quite small concentrations of the rare earth solutions can produce large effects on colloidal materials which present all the other characters of emulsoids. Many experiments on a variety of colloidal solutions lead me to the conclusion that a fairer statement of the characteristic relations between emulsoid colloids and electrolytes would be this: that as compared with the suspensoids there is a very great difference in the relative concentrations of simple di- and tri-valent ions needed to produce the same effect. While for suspensoids the ratio of the activities of di- to tri-valent ions is taken ordinarily as of the order of 3 to 100 , for emulsoids it is rather of the order of 1 to 10,000 . It is certain that these ratios vary widely with different colloids, and that from a more precise study of these ratios than has at present been undertaken much more will be learnt about the colloids themselves, but the broad distinction appears to hold.

On the artificial scheme of membranes, then, magnesium produces effects tending in the same direction as those produced by the simple trivalent ions and by the hydrogen ion, but is enormously less potent than these.

Precisely the same is true of the action of $\mathrm{Mg} \cdots$ on the heart.

* Incidentally it should be noted that the effect of hydrolysis, as regards the activity of the solution on the heart and on colloids such as gelatine, is precisely opposite in the case of these solutions of divalent ions from that described for trivalent ions. For the $\mathrm{H}$ ions, though less powerful than the equivalent concentration of simple trivalent ions, are much more powerful than the equivalent concentration of divalent ions in these relations. Consequently a solution of beryllium, which is hydrolyzed, affects the heart, and colloid systems of the class sensitive to $\mathrm{H} \cdot$, much more powerfully than the non-hydrolyzed solutions of magnesium. 
In the ray heart, for instance, a concentration of $000001 \mathrm{M}$ to $.00001 \mathrm{M}$ of $\mathrm{Ce} \cdots$ or $\mathrm{Nd} \cdots$ is sufficient to cause prompt arrest in diastole. The same effect can be produced by $\mathrm{Mg} \cdot$, but the concentration needed is about $02 \mathrm{M}$. If our explanation of the action of magnesium on the heart is true, it should of course be found that the dose of $\mathrm{Mg}$ needed to arrest the heart depends greatly on the $\mathrm{P}+$ of the solution in which it is applied. An increase in the

$\mathrm{H}$

numerical value of the $\mathrm{P}+$ should tend to counterbalance the effect $\mathrm{H}$

of magnesium. This is abundantly confirmed by experiment. For example : a ray heart was brought to rest by raising the concentration of $\mathrm{Mg} \cdot$ in the solution, perfusing it to $025 \mathrm{M}$. The diastolic arrest occurred after two or three contractions. When the heart had remained completely at rest for a minute with this solution running through, without changing the concentration of $\mathrm{Mg} \cdot$ the $\mathrm{P}+$ of

the solution was raised from about 6.5 to 9 . Within a few seconds the beart started beating, and continued to beat in this solution as strongly and regularly as in the original solution which contained no excess of $\mathrm{Mg}$ '

Consideration of the relations of colloidal materials to simple and complex trivalent kations enables us to make certain inferences as to the nature of these colloidal substances from which we can predict their relations to certain other ions. Applying this principle to the heart, we arrive at conclusions as to the nature of the colloidal membranes in its structure from which we can predict the action of magnesium upon the heart-beat.

Finally, I wish to indicate certain directions in which experiments of this kind may assist in the development of a scientific comparative physiology.

Two instances which I have encountered in the course of work at the Plymouth Laboratory this summer may serve as illustrations.*

The facts already stated about the general similarity in the relations of the heart muscle of widely different species to $\mathrm{Ca}, \mathrm{Sr}$, and $\mathrm{Ba}$ encourage the belief that the general character of the mechanism is the same in these varieties of heart muscle. Can we find differences in the relations of the membranes to "charging" electrolytes? What kind of differences are to be looked for?

A study of the properties of colloids by a variety of methods leads to the following conclusions with respect to the part played by the

* I am much indebted to Miss Dale, of Newnham College, for her skilful assistance in this work.

NEW SERIES.-VOL. IX. NO. 2. OCTOBER, 1911. 
nature of the colloid itself in determining its relations to electrolytes.

(1) Different surfaces in the same solution may take on different electrical charges.

(2) Different surfaces possessing the same charge in some particular solution may require different concentrations of the same electrolyte to produce equal changes in their charges.

The physiological expression of a difference in the heart membranes of the kind indicated in the first of these conclusions would be of this type: that one kind of heart should behave in a neutral solution as another would behave in an acid or in an alkaline solution, other conditions being equal.

The heart of the mollusc Pecten maximus differs from all the vertebrate hearts studied in this way. On perfusing it with "neutral saline" it stops instantly in systole. It will beat, however, if we alter the $\mathrm{P}+$ of

the solution in the direction of slightly increased acidity; if the change is carried a little further, diastolic arrest-the characteristic acid effect -is then produced. Now the $\mathrm{P}+$ of the solution which perfuses the

$\mathrm{H}$

heart of the living Pecten-the blood of the animal-is about 6.5 to 7 , that is to say, very slightly on the alkaline side of our "neutral saline." How then can the heart beat in such a solution? The answer is simple. The blood of Pecten contains a large concentration of magnesium. The divalent $\mathrm{Mg} \cdot$ is present in sufficient concentration to bring the charge of the membranes to the value required for their proper differential permeability.

The assertion that the blood of Pecten is practically the same as seawater overlooks one important fact about it. Though its osmotic pressure and concentrations of $\mathrm{Na}, \mathrm{Mg}, \mathrm{K}, \mathrm{Ca}$, etc. are closely the same as in sea-water, there is a distinct difference in the $\mathrm{P}+$ of the two

$\mathrm{H}$

fluids. The $\mathrm{P}+$ of sea-water varies from about $7 \cdot 9$ to $8 \cdot 3$, while that $\mathrm{H}$

of the Pecten blood is rather below 7. The difference may be demonstrated by adding a drop of a solution of rosolic acid to a little seawater and to a similar quantity of the colourless blood of Pecten. The colour given by the former is bright pink, that of the latter yellow or yellowish pink.

Thus we find that sea-water, despite its high concentration of magnesium, on perfusion instantly stops the Pecten heart in systole. 
But if by the addition of a little dilute hydrochloric acid we change the $\mathrm{P}+$ of the sea-water to the same value as that found for the blood

$\mathrm{H}$

of Pecten (about 1.5 cc. of $1 \mathrm{M} \mathrm{HCl}$ in $100 \mathrm{cc}$. of sea-water), we have at once a solution which sustains the beat of the heart admirably. A solution of the same composition as this but without magnesium causes the heart to stop in systole. Further, as is predicted theoretically, it is possible to replace the $\mathrm{Mg}$ by a very small concentration of a simple trivalent ion. Thus the Pecten heart which has stopped beating in "neutral saline" can be made to beat again and be kept beating for a considerable time by the addition to the solution of a concentration of about $\cdot 00003 \mathrm{M}$ of $\mathrm{Ce} \cdots$ or $\mathrm{Nd} \cdots$. Here we have one trivalent ion doing the work of some 40,000 divalent ions, and it is therefore not surprising that the solution with rare earth does not, as a rule, keep the heart going so regularly as the solution with magnesium. Higher concentrations of the simple trivalent ions (e.g. $.0001 \mathrm{M}$ ) stop the Pecten heart in diastole exactly in the same way as a too acid solution.

We may express the difference between the heart of Pecten and the hearts of the vertebrates which have been studied, by saying that the membranes have different iso-electric points. This kind of difference is most probably related to a difference in the chemical composition of the membranes; it being a well-established fact that different protein substances found in the animal kingdom do show differences of this kind, according to the predominance of the "acidic" or " basic" aminoacids included in their composition.

It is by no means a new suggestion that the differences between different species are at root differences in the chemical composition of their tissues; but as far as I am aware it has not before been pointed out by what mechanism such differences can affect the physiological behaviour of the living heart.

The second conclusion formulated above as to the relations between different surfaces and the same electrolytes finds biological application in the differentiation of more closely allied species.

The hearts of the elasmobranchs Raia and Scyllium are kept beating satisfactorily for many hours by perfusion with the same "neutral" solution containing sodium, potassium, calcium, and magnesium chlorides and urea, the solution being thoroughly aerated and of $\mathrm{P}+$

about 6.5. The concentration of $\mathrm{Mg}$ in the solution generally used was $.005 \mathrm{M}$. Removal of the $\mathrm{Mg}$ from the solution only causes the beats to get rather faster. Increase of the concentration of magnesium 
causes slowing of the heart-beats, and if carried far enough, stoppage. Qualitatively these phenomena are alike in the hearts of the ray and of the dogfish. But studied quantitatively the ray heart is found to be much more sensitive to $\mathrm{Mg}$ than is the dogfish heart. Thus taking the mean of a number of observations on ray* hearts, the concentration needed to give a 50 per cent reduction in the rate of beat (calling the rate in the solution with $005 \mathrm{M} \mathrm{Mg}=100$ ) was only 009 M. For Scyllium it was 05 M. To stop the ray heart the concentration of $\mathrm{Mg}$ ' needed was about $02 \mathrm{M}$, while to stop the heart of Scyllium a concentration usually greater than $1 \mathrm{M}$ was required.

A corresponding difference is found in the sensitiveness of these hearts to the simple trivalent ions. Thus $00001 \mathrm{M}$. Ce $\cdots$ readily stops the heart of Raia, while a concentration approaching ten times this magnitude is needed to produce the same effect on the heart of Scyllium.

In these respects the heart of the angel fish, Rhina squatina, was found to resemble that of the dogfish rather than that of the ray. The curve representing its behaviour in the presence of various concentrations of $\mathrm{Mg}$ lies between that plotted for Raia and that for Scyllium, but much nearer the latter than the former. This is interesting, as in the ordinary morphological classification Rhina is placed between the Scylliidae and the Raiidae. It is said that Rhina resembles rays rather than dogfish in its general habits, yet it is possible that the character indicated by these experiments represents something deeper seated than the details of the responses given by the central nervous system.

It is at any rate conceivable that by the extension of work along these lines we may learn to express genetic affinity in physico-chemical terms.

One further point. It will be recollected that the behaviour of a membrane depends not only on its original composition and on the nature of the solution immediately surrounding it, but also to some extent on its previous history. I have found that the blood plasma of Scyllium and of Rhina is of $\mathrm{P}+$ numerically slightly higher than $\mathrm{H}$

the plasma of Raia (clavata or blanda). It is possible that the difference in sensitiveness of the hearts of these animals to the

* Two species, $R$. clavata and $R$. blanda, were used in these experiments. No difference could be detected in the behaviour of the hearts of these two species towards electrolytes. Using rays of very different sizes no constant differences were found between the large and the small specimens. 
agencies we have named is to be explained in part by the fact that their membranes have been bathed for years before the experiment in solutions of slightly different $\mathrm{P}+$.

\section{$\mathrm{H}$}

There are striking differences in the relations of the hearts of different species of animals to hydrogen ion concentration and to polyvalent ions: these correspond exactly to differences found in the effects of these ions on the ionic permeability of different colloidal materials. A mechanism is thus suggested which explains how differences in the chemical constitution of a tissue component may cause differences in the physiological behaviour of the living tissue.

I venture to hope that these fragmentary remarks will make it apparent that a field of research lies open here, containing treasure not alone for the physiologist who desires to elucidate the mechanisms by which vital processes are wrought in the individual, but also for the zoologist, who in looking for clues of genetic affinities has learnt the limitations of pure morphology and the truth of the axiom of physiology that by studying what a cell can do we shall best learn what it is.

For the furtherance of the science of Comparative Physiology the co-operation of morphologists and physiologists with physical, organic, analytical, and colloid chemists is necessary. This country is not lacking in any of these. The study of the science can be carried on best where the greatest variety of animals, living and in good condition, can be provided. This condition is most perfectly fulfilled at a marine station.

At Plymouth the fauna is rich, and the facilities provided in the Laboratory for keeping animals in condition for physiological experiment are admirable. It is earnestly to be wished that more workers in this country would interest themselves in a branch of zoology and of physiology pregnant with interest for both sciences, and that their efforts might secure for the Plymouth Laboratory permanent accommodation and equipment for physiological work, on a scale approaching that provided by marine stations in other countries.

In conclusion, I would express my warmest thanks to the Director and Staff of the Laboratory of the Marine Biological Association at Plymouth for much valuable assistance and advice.*

September, 1911.

* I am indebted to Professor Stanley Gardiner for the use of a University Table at the Plymouth Laboratory during July, August, and part of September.

The expenses of the researches were defrayed in part by a grant from the Government Grant Committee of the Royal Society. 


\section{BIBLIOGRAPHY.}

References to work on the subjects named below will be found in the following:-

Measurement of hydrogen ion concentration, etc. :

Sörensen, C. R. Lab., Carlsberg, VIII, p. 1. 1909.

Palitzsch, Publ. de Circonstance, No. 60. Cons. perm. pour l'explor. de la mer. 1911.

Action of chemical substances on the heart:

Langendorff, Ergebnisse d. Physiol., IV.

Luciani, Human Physiology, trans. by Welby, p. 337. 1911.

Electric charge of membranes:

Perrin, Journ. de Chimie Physique, II, p. 601. 1904.

Action of trivalent ions on heart and on colloids :

Mines, Journ. Physiol., XL, p. 327. 1910. Ibid., XLII, p. 309. 1911. 